Supplemental Information

\title{
Refining the N-termini of the SARS-CoV-2 Spike Protein and its Receptor Binding Domain
}

\author{
Robert A. D'Ippolito ${ }^{1}$, Matthew R. Drew ${ }^{1}$, Jennifer Mehalko ${ }^{1}$, Kelly Snead ${ }^{1}$, Vanessa \\ Wall $^{1}$, Zoe Putman ${ }^{1}$, Dominic Esposito ${ }^{1}$, Caroline J. DeHart ${ }^{1 *}$ \\ ${ }^{1} \mathrm{NCI}$ RAS Initiative, Cancer Research Technology Program, Frederick National \\ Laboratory for Cancer Research, Frederick, MD 21702, USA \\ *Corresponding Author: caroline.dehart@nih.gov
}

\section{Summary}

Figure S1: Structural features of the Constructs Analyzed. Figure S2: SDS-PAGE image of All Constructs.

Figure S3: The Predicted Mt. Sinai RBD Protein Sequence.

Figure S4: The Predicted Ragon RBD Protein Sequence.

Figure S5: The Predicted M69 RBD Protein Sequence.

Figure S6: ReSpect ${ }^{\mathrm{TM}}$ deconvolution of initial RBD constructs.

Figure S7: Intact Mass Resolution Comparison of M68

Figure S8: The Predicted M96 RBD Protein Sequence.

Figure S9: Comparative ELISA sensitivity of the four RBD constructs.

Table S1. Intact Mass Analysis of the Original Mt. Sinai Construct.

Table S2. Intact Mass Analysis of M67.

Table S3. Intact Mass Analysis of the Original Ragon Construct.

Table S4. Intact Mass Analysis of M68.

Table S5. Intact Mass Analysis of M69.

Table S6. Intact Mass Analysis of M98. 


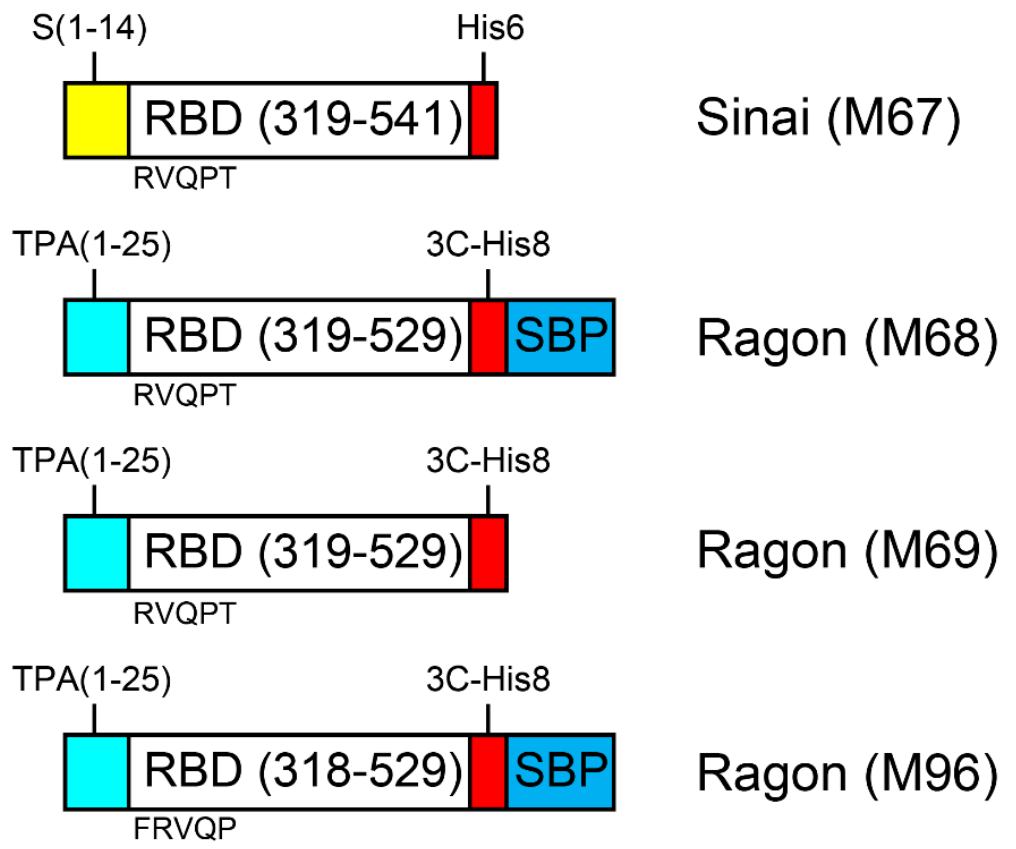

Figure S1. Structural features of the constructs analyzed. M67: S leader, Sinai 319-541, C-term His6 [Figure S3]. M68: TPA leader, Ragon 319-529, C-term 3C-His8-SBP [Figure S4]. M69: TPA leader, Ragon 319-529, C-term His8 [Figure S5]. M96: TPA leader, Ragon 318-529, C-term 3C-His8-SBP [Figure S8].

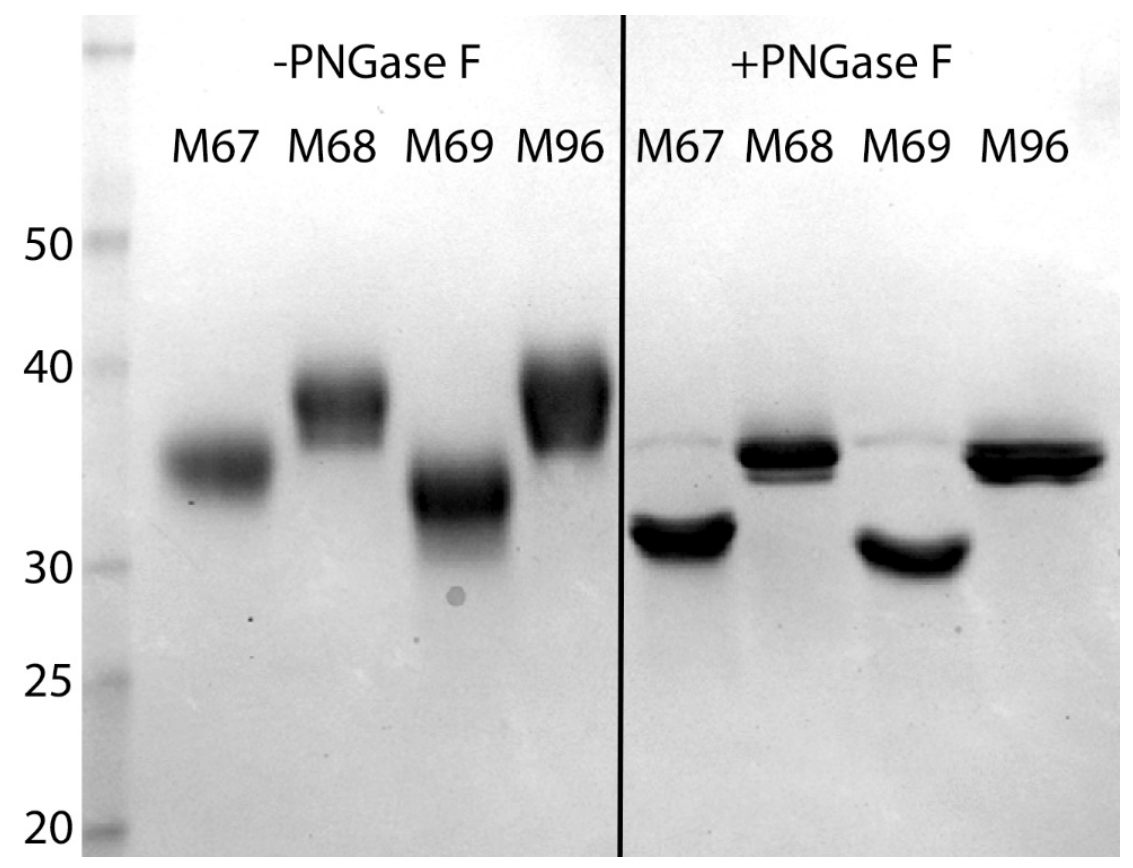

Figure S2. SDS-PAGE image of all constructs. 


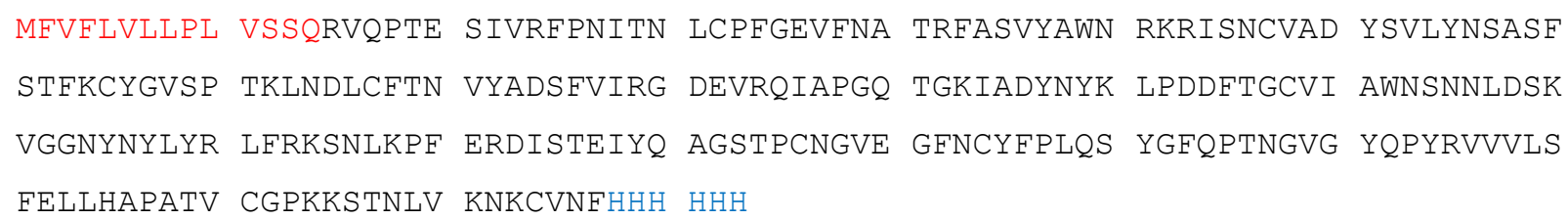

Figure S3. The predicted Mt. Sinai RBD protein sequence from the construct DNA. Red: M1-Q14 of fulllength spike signal sequence. Black: R319-F541; RBD of spike. Blue: His6 tag.

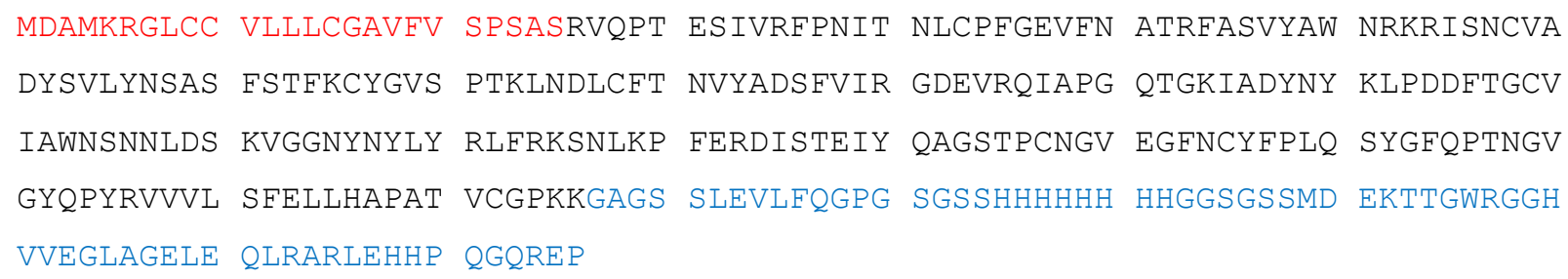

Figure S4. The predicted Ragon RBD protein sequence from the construct DNA. Red: Tissue plasminogen activator (TPA) signal sequence. Black: R319-K529; RBD of spike. Blue: HRV3C protease cleavage site, His8, streptavidin-binding peptide combination tag.

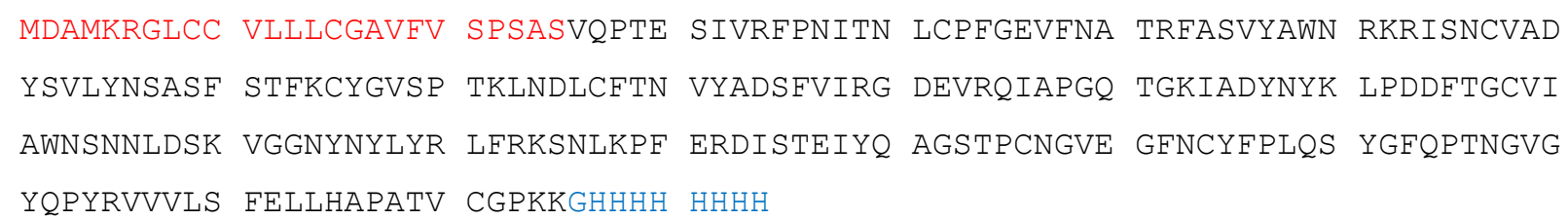

Figure S5. The predicted M69 RBD protein sequence from the construct DNA. Red: Tissue plasminogen activator (TPA) signal sequence. Black: V320-K529; RBD of spike. Blue: His8 tag. 

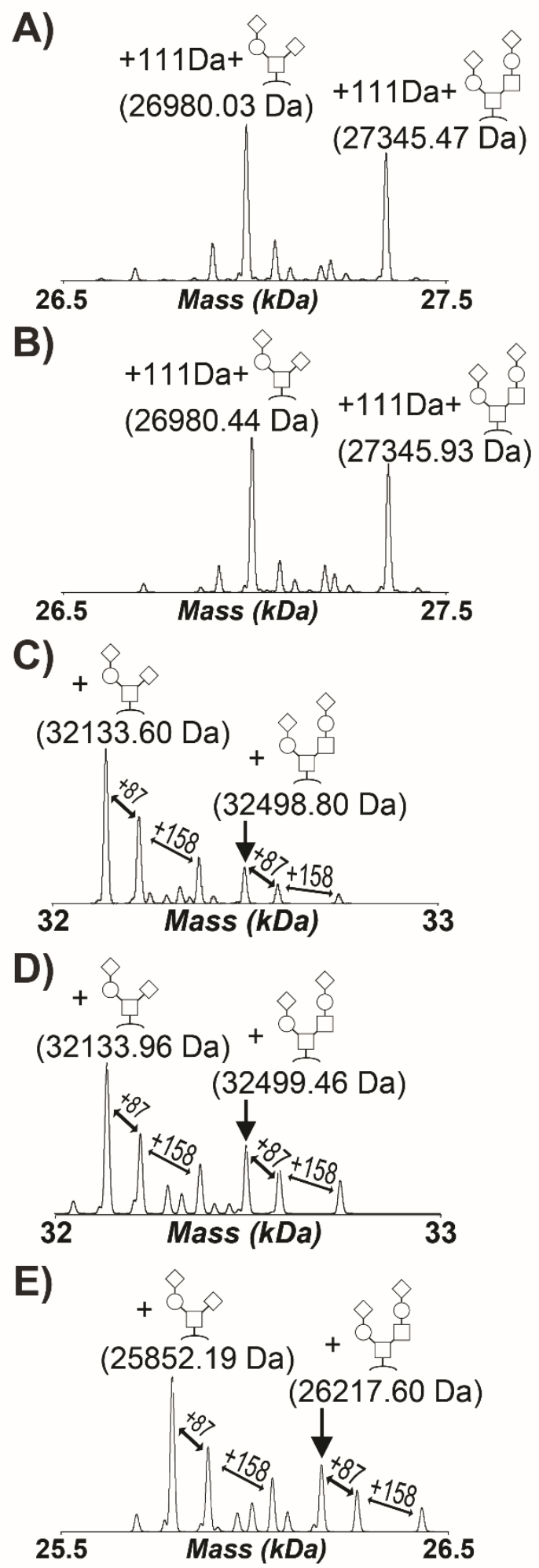

Figure S6. ReSpect ${ }^{\mathrm{TM}}$ deconvolution of initial RBD constructs. A) Original Mt. Sinai Construct. B) M67, the FNL vector optimized version of the Mt. Sinai construct. C) Original Ragon Construct. D) M68, the FNL vector optimized version of the Ragon Construct. E) M69, the FNL vector optimized version of the Ragon Construct with only a His8 C-terminal tag. 


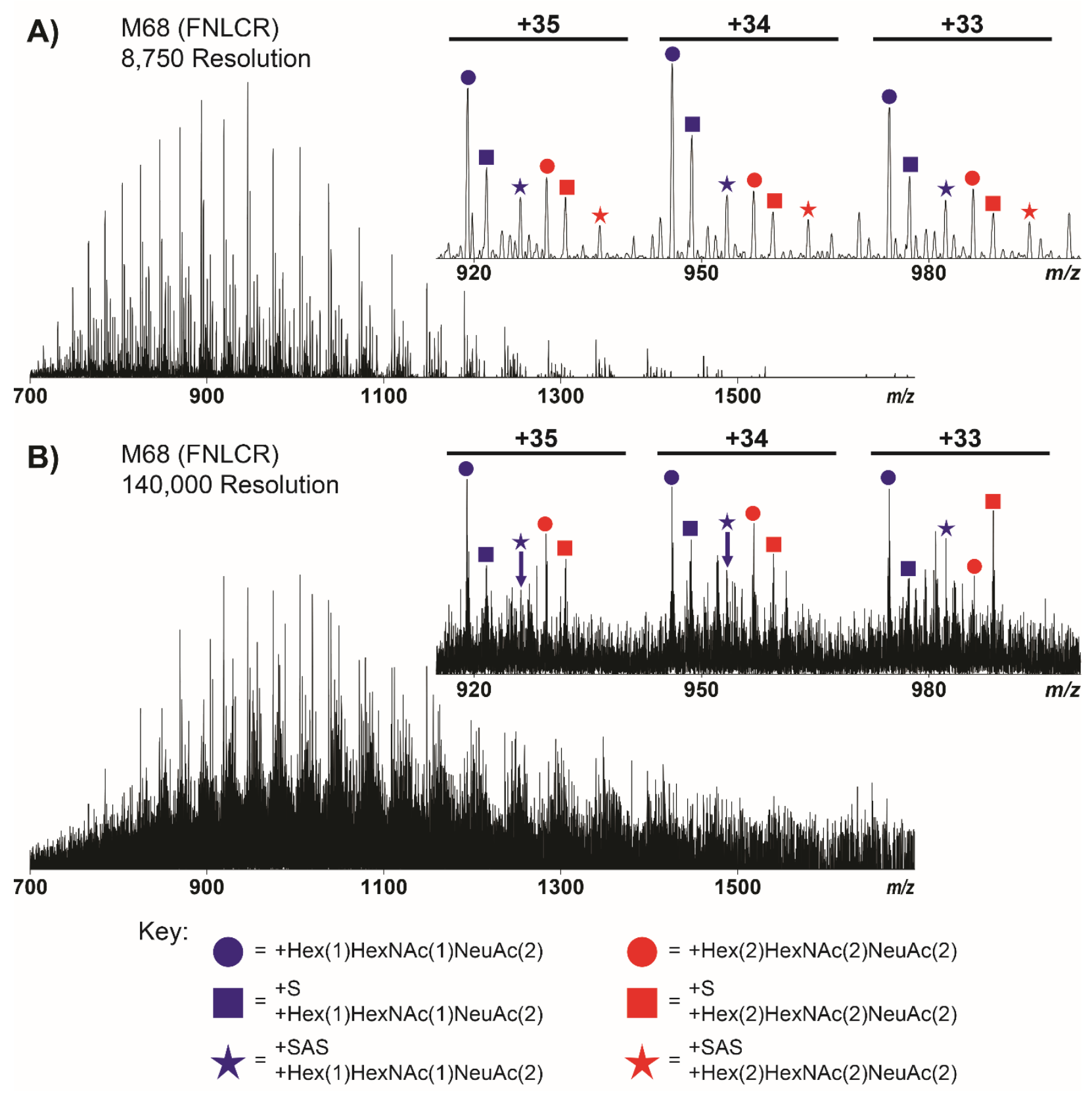

Figure S7: Intact Mass Resolution Comparison of M68. A) Intact mass spectrum of M68 following PNGaseF treatment with an FT resolution of 8,750. B) Intact mass spectrum of M68 following PNGaseF treatment with an FT resolution of 140,000. The insets contain an expansion of $915-1000 \mathrm{~m} / \mathrm{z}$, corresponding to charge states $+33,+34$, and +35 , to highlight spectral complexity at the given FT resolution. 


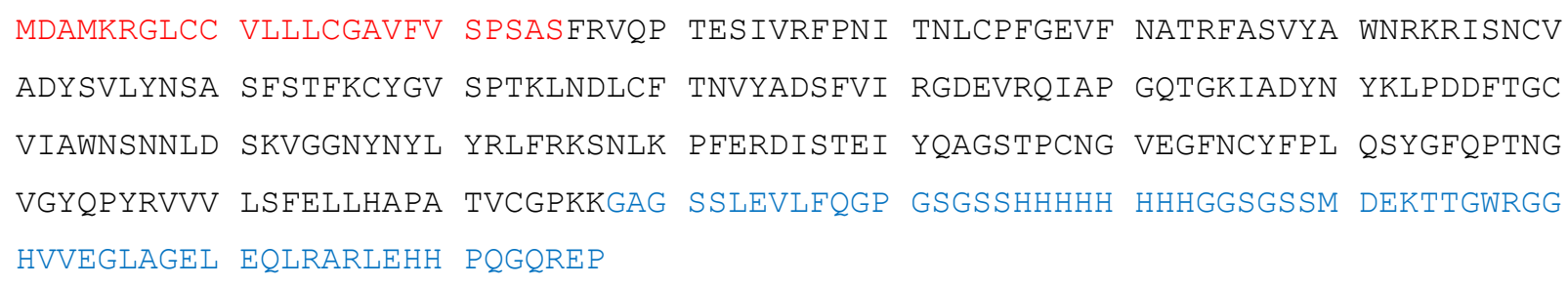

Figure S8. The predicted M96 RBD protein sequence from the construct DNA. Red: Tissue plasminogen activator (TPA) signal sequence. Black: F318-K529; RBD of spike. Blue: HRV3C protease cleavage site, His8, streptavidin-binding peptide combination tag.

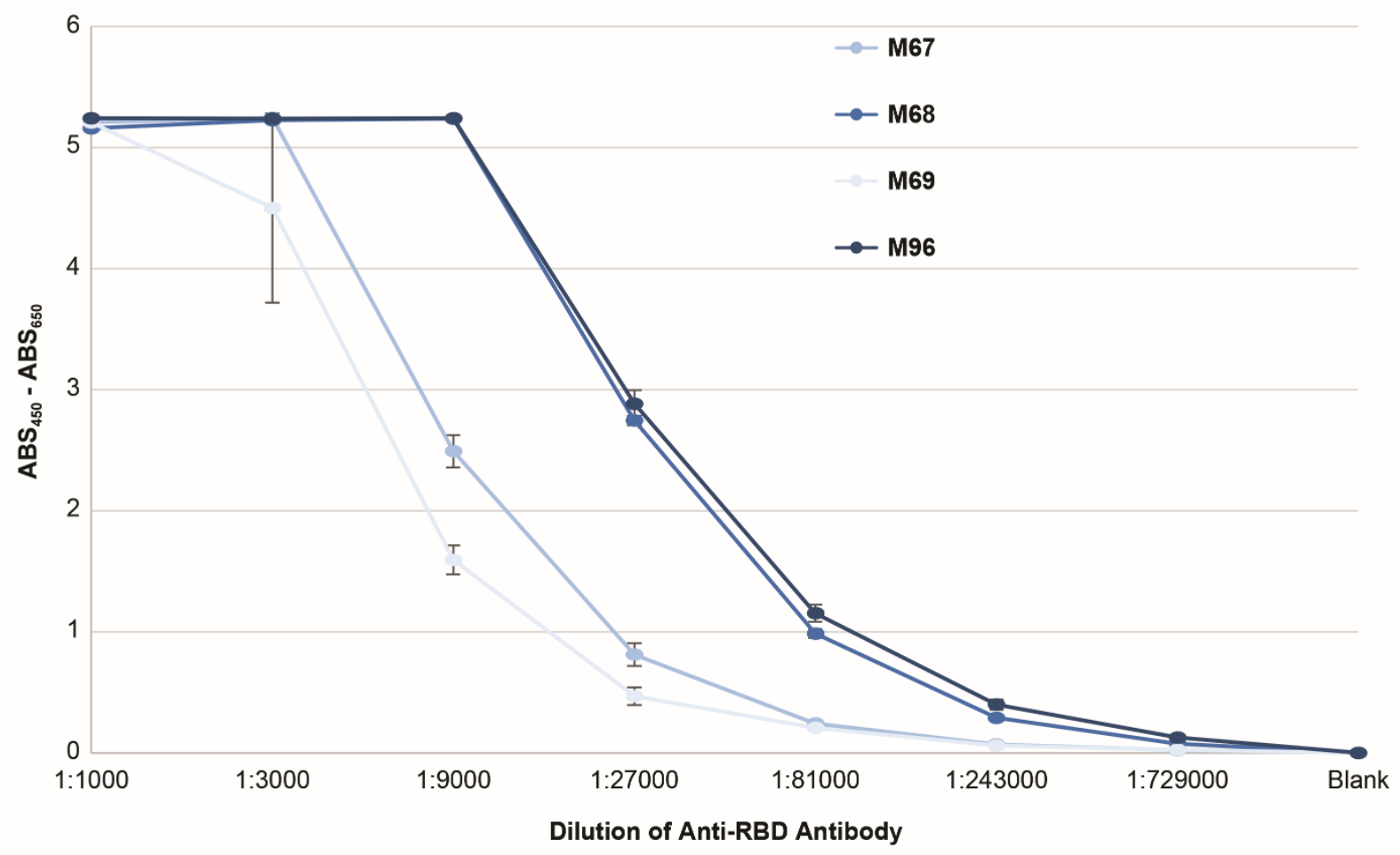

Figure S9. Comparative ELISA sensitivity of the four RBD constructs. ELISA was performed with the previous (M67, M68, and M69) and newest (M96) RBD constructs coating the ELSIA plates and treated with anti-RBD monoclonal antibodies at the reported dilutions (X-axis). Absorbance measurements were taken at $450 \mathrm{~nm}$ which were corrected by subtraction with the absorbance at $650 \mathrm{~nm}$ (Y-axis). Triplicate measurements were performed with error bars equal to the standard deviation. 
Table S1. Intact mass analysis of the original Mt. Sinai construct.

\begin{tabular}{|c|c|c|c|c|c|c|}
\hline Proteoform & $\begin{array}{c}\text { Theoretical } \\
\text { Average } \\
\text { Mass (Da) }\end{array}$ & $\begin{array}{c}\text { Observed } \\
\text { Average } \\
\text { Mass } \\
\text { (Da) }\end{array}$ & $\begin{array}{c}\text { Average } \\
\text { Mass } \\
\text { Error } \\
\text { (Da) }\end{array}$ & Intensity & $\begin{array}{c}\text { Relative } \\
\text { Abundance }\end{array}$ & $\begin{array}{c}\text { Fractional } \\
\text { Abundance }\end{array}$ \\
\hline $\begin{array}{c}+ \text { pyroGlu } \\
+\mathrm{Hex}(1) \mathrm{HexNAc}(1) \mathrm{NeuAc}(2)\end{array}$ & 26980.19 & 26980.03 & 0.16 & $4.25 \mathrm{E}+08$ & $100.00 \%$ & $54.18 \%$ \\
\hline $\begin{array}{c}+ \text { pyroGlu } \\
+\mathrm{Hex}(2) \mathrm{HexNAc}(2) \mathrm{NeuAc}(2)\end{array}$ & 27345.52 & 27345.47 & 0.05 & $3.59 \mathrm{E}+08$ & $84.59 \%$ & $45.82 \%$ \\
\hline
\end{tabular}

Table S2. Intact mass analysis of M67.

\begin{tabular}{|c|c|c|c|c|c|c|c|c|c|}
\hline Proteoform & $\begin{array}{l}\text { Theoretical } \\
\text { Monoisotopic } \\
\text { Mass (Da) }\end{array}$ & $\begin{array}{c}\text { Observed } \\
\text { Monoisotopic } \\
\text { Mass (Da) }\end{array}$ & $\begin{array}{l}\text { Monoisotopic } \\
\text { Mass Error } \\
\text { (Da) }\end{array}$ & $\begin{array}{l}\text { Theoretical } \\
\text { Average } \\
\text { Mass (Da) }\end{array}$ & $\begin{array}{c}\text { Observed } \\
\text { Average } \\
\text { Mass } \\
\text { (Da) }\end{array}$ & $\begin{array}{c}\text { Average } \\
\text { Mass } \\
\text { Error } \\
\text { (Da) }\end{array}$ & Intensity & $\begin{array}{l}\text { Relative } \\
\text { Abundance }\end{array}$ & $\begin{array}{l}\text { Fractional } \\
\text { Abundance }\end{array}$ \\
\hline 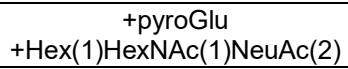 & 26963.087 & 26964.117 & 1.030 & 26980.19 & 26980.44 & 0.25 & $5.63 \mathrm{E}+08$ & $100.00 \%$ & $55.86 \%$ \\
\hline 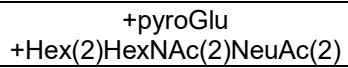 & 27328.219 & 27329.218 & 0.999 & 27345.52 & 27345.93 & 0.41 & $4.45 \mathrm{E}+08$ & $79.02 \%$ & $44.14 \%$ \\
\hline
\end{tabular}

Table S3. Intact mass analysis of the original Ragon construct.

\begin{tabular}{|c|c|c|c|c|c|c|}
\hline Proteoform & $\begin{array}{l}\text { Theoretical } \\
\text { Average } \\
\text { Mass (Da) }\end{array}$ & $\begin{array}{c}\text { Observed } \\
\text { Average } \\
\text { Mass } \\
\text { (Da) }\end{array}$ & $\begin{array}{c}\text { Average } \\
\text { Mass } \\
\text { Error } \\
\text { (Da) }\end{array}$ & Intensity & $\begin{array}{l}\text { Relative } \\
\text { Abundance }\end{array}$ & $\begin{array}{l}\text { Fractional } \\
\text { Abundance }\end{array}$ \\
\hline$+\mathrm{Hex}(1) \mathrm{HexNAc}(1) \mathrm{NeuAc}(2)$ & 32133.64 & 32133.60 & 0.04 & $1.87 \mathrm{E}+08$ & $100.00 \%$ & $42.10 \%$ \\
\hline $\begin{array}{c}+\mathrm{S} \\
+\mathrm{Hex}(1) \mathrm{HexNAc}(1) \mathrm{NeuAc}(2)\end{array}$ & 32220.72 & 32220.73 & 0.01 & $1.10 \mathrm{E}+08$ & $58.90 \%$ & $24.80 \%$ \\
\hline $\begin{array}{c}+\mathrm{SAS} \\
+\mathrm{Hex}(1) \mathrm{HexNAc}(1) \mathrm{NeuAc}(2)\end{array}$ & 32378.88 & 32378.78 & 0.10 & $6.13 \mathrm{E}+07$ & $32.81 \%$ & $13.81 \%$ \\
\hline$+\mathrm{Hex}(2) \mathrm{HexNAc}(2) \mathrm{NeuAc}(2)$ & 32498.98 & 32498.80 & 0.18 & $4.28 \mathrm{E}+07$ & $22.92 \%$ & $9.65 \%$ \\
\hline $\begin{array}{c}+\mathrm{S} \\
+\mathrm{Hex}(2) \mathrm{HexNAc}(2) \mathrm{NeuAc}(2)\end{array}$ & 32586.06 & 32585.26 & 0.80 & $2.64 \mathrm{E}+07$ & $14.13 \%$ & $5.95 \%$ \\
\hline $\begin{array}{c}+\mathrm{SAS} \\
+\mathrm{Hex}(2) \mathrm{HexNAc}(2) \mathrm{NeuAc}(2)\end{array}$ & 32744.22 & 32744.87 & 0.65 & $1.64 \mathrm{E}+07$ & $8.77 \%$ & $3.69 \%$ \\
\hline
\end{tabular}

Table S4. Intact mass analysis of M68.

\begin{tabular}{|c|c|c|c|c|c|c|c|c|c|}
\hline Proteoform & $\begin{array}{l}\text { Theoretical } \\
\text { Monoisotopic } \\
\text { Mass (Da) }\end{array}$ & $\begin{array}{l}\text { Observed } \\
\text { Monoisotopic } \\
\text { Mass (Da) }\end{array}$ & $\begin{array}{l}\text { Monoisotopic } \\
\text { Mass Error } \\
\text { (Da) }\end{array}$ & $\begin{array}{c}\text { Theoretical } \\
\text { Average } \\
\text { Mass (Da) }\end{array}$ & $\begin{array}{c}\text { Observed } \\
\text { Average } \\
\text { Mass } \\
\text { (Da) }\end{array}$ & $\begin{array}{c}\text { Average } \\
\text { Mass } \\
\text { Error } \\
\text { (Da) }\end{array}$ & Intensity & $\begin{array}{l}\text { Relative } \\
\text { Abundance }\end{array}$ & $\begin{array}{l}\text { Fractional } \\
\text { Abundance }\end{array}$ \\
\hline$+\mathrm{Hex}(1) \mathrm{HexNAc}(1) \mathrm{NeuAc}(2)$ & 32113.531 & 32114.634 & 1.103 & 32133.64 & 32133.96 & 0.32 & $2.37 \mathrm{E}+08$ & $100.00 \%$ & $34.13 \%$ \\
\hline $\begin{array}{c}+\mathrm{S} \\
+\mathrm{Hex}(1) \mathrm{HexNAc}(1) \mathrm{NeuAc}(2)\end{array}$ & 32200.563 & 32200.635 & 0.072 & 32220.72 & 32221.42 & 0.70 & $1.26 \mathrm{E}+08$ & $53.32 \%$ & $18.19 \%$ \\
\hline $\begin{array}{c}\text { +SAS } \\
+\mathrm{Hex}(1) \mathrm{HexNAc}(1) \mathrm{NeuAc}(2) \\
\end{array}$ & 32358.638 & $N / D^{\ddagger}$ & N/D & 32378.88 & 32378.76 & 0.12 & $8.49 \mathrm{E}+07$ & $35.86 \%$ & $12.24 \%$ \\
\hline$+\mathrm{Hex}(2) \mathrm{HexNAc}(2) \mathrm{NeuAc}(2)$ & 32478.663 & 32478.711 & 0.048 & 32498.98 & 32499.46 & 0.48 & $1.09 \mathrm{E}+08$ & $46.19 \%$ & $15.76 \%$ \\
\hline $\begin{array}{c}+\mathrm{S} \\
+\mathrm{Hex}(2) \mathrm{HexNAc}(2) \mathrm{NeuAc}(2)\end{array}$ & 32565.695 & N/D & N/D & 32586.06 & 32586.02 & 0.04 & $8.20 \mathrm{E}+07$ & $34.62 \%$ & $11.81 \%$ \\
\hline $\begin{array}{c}+\mathrm{SAS} \\
+\mathrm{Hex}(2) \mathrm{HexNAc}(2) \mathrm{NeuAc}(2)\end{array}$ & 32723.764 & N/D & $N / D$ & 32744.22 & 32745.24 & 1.02 & $5.46 \mathrm{E}+07$ & $23.05 \%$ & $7.87 \%$ \\
\hline
\end{tabular}

\footnotetext{
$\mp \mathrm{N} / \mathrm{D}=$ not detected in deconvolution
} 
Table S5. Intact mass analysis of M69.

\begin{tabular}{|c|c|c|c|c|c|c|c|c|c|}
\hline Proteoform & $\begin{array}{l}\text { Theoretical } \\
\text { Monoisotopic } \\
\text { Mass (Da) }\end{array}$ & $\begin{array}{c}\text { Observed } \\
\text { Monoisotopic } \\
\text { Mass (Da) }\end{array}$ & $\begin{array}{l}\text { Monoisotopic } \\
\text { Mass Error } \\
\text { (Da) }\end{array}$ & $\begin{array}{l}\text { Theoretical } \\
\text { Average } \\
\text { Mass (Da) }\end{array}$ & $\begin{array}{c}\text { Observed } \\
\text { Average } \\
\text { Mass } \\
\text { (Da) } \\
\end{array}$ & $\begin{array}{c}\text { Average } \\
\text { Mass } \\
\text { Error } \\
\text { (Da) }\end{array}$ & Intensity & $\begin{array}{l}\text { Relative } \\
\text { Abundance }\end{array}$ & $\begin{array}{l}\text { Fractional } \\
\text { Abundance }\end{array}$ \\
\hline$+\mathrm{Hex}(1) \mathrm{HexNAc}(1) \mathrm{NeuAc}(2)$ & 25835.497 & 25836.543 & 1.046 & 25851.84 & 25852.19 & 0.35 & $2.47 \mathrm{E}+08$ & $100.00 \%$ & $35.60 \%$ \\
\hline $\begin{array}{c}+\mathrm{S} \\
+\mathrm{Hex}(1) \mathrm{HexNAc}(1) \mathrm{NeuAc}(2)\end{array}$ & 25922.529 & 25922.606 & 0.077 & 25938.92 & 25939.49 & 0.57 & $1.39 \mathrm{E}+08$ & $56.34 \%$ & $20.05 \%$ \\
\hline $\begin{array}{c}+\mathrm{SAS} \\
+\mathrm{Hex}(1) \mathrm{HexNAc}(1) \mathrm{NeuAc}(2) \\
\end{array}$ & 26080.599 & 26080.615 & 0.016 & 26097.08 & 26097.34 & 0.26 & $8.58 \mathrm{E}+07$ & $34.73 \%$ & $12.36 \%$ \\
\hline$+\mathrm{Hex}(2) \mathrm{HexNAc}(2) \mathrm{NeuAc}(2)$ & 26200.630 & 26200.652 & 0.022 & 26217.18 & 26217.60 & 0.42 & $1.08 \mathrm{E}+08$ & $43.77 \%$ & $15.58 \%$ \\
\hline $\begin{array}{c}+\mathrm{S} \\
+\mathrm{Hex}(2) \mathrm{HexNAc}(2) \mathrm{NeuAc}(2)\end{array}$ & 26287.662 & 26287.675 & 0.013 & 26304.26 & 26304.88 & 0.62 & $6.74 \mathrm{E}+07$ & $27.30 \%$ & $9.72 \%$ \\
\hline $\begin{array}{c}+\mathrm{SAS} \\
+\mathrm{Hex}(2) \mathrm{HexNAc}(2) \mathrm{NeuAc}(2)\end{array}$ & 26245.731 & $N / D^{\ddagger}$ & $\mathrm{N} / \mathrm{D}$ & 26462.42 & 26463.21 & 0.79 & $4.64 \mathrm{E}+07$ & $18.79 \%$ & $6.69 \%$ \\
\hline
\end{tabular}

$\mp \mathrm{N} / \mathrm{D}=$ not detected in deconvolution

Table S6. Intact mass analysis of M98.

\begin{tabular}{|c|c|c|c|c|c|c|c|c|c|}
\hline Proteoform & $\begin{array}{l}\text { Theoretical } \\
\text { Monoisotopic } \\
\text { Mass (Da) }\end{array}$ & $\begin{array}{l}\text { Observed } \\
\text { Monoisotopic } \\
\text { Mass (Da) }\end{array}$ & $\begin{array}{l}\text { Monoisotopic } \\
\text { Mass Error } \\
\text { (Da) }\end{array}$ & $\begin{array}{l}\text { Theoretical } \\
\text { Average } \\
\text { Mass (Da) }\end{array}$ & $\begin{array}{c}\text { Observed } \\
\text { Average } \\
\text { Mass } \\
\text { (Da) } \\
\end{array}$ & $\begin{array}{c}\text { Average } \\
\text { Mass } \\
\text { Error } \\
\text { (Da) } \\
\end{array}$ & Intensity & $\begin{array}{c}\text { Relative } \\
\text { Abundance }\end{array}$ & $\begin{array}{l}\text { Fractional } \\
\text { Abundance }\end{array}$ \\
\hline Unmodified & 31313.276 & 31313.312 & 0.036 & 31332.98 & 31332.53 & 0.45 & $2.61 \mathrm{E}+07$ & $31.17 \%$ & $15.16 \%$ \\
\hline$+\mathrm{Hex}(1) \mathrm{HexNAc}(1) \mathrm{NeuAc}(2)$ & 32260.599 & 32260.676 & 0.077 & 32280.82 & 32280.64 & 0.18 & $8.38 \mathrm{E}+07$ & $100.00 \%$ & $48.62 \%$ \\
\hline$+\mathrm{Hex}(2) \mathrm{HexNAc}(2) \mathrm{NeuAc}(2)$ & 32625.732 & 32626.786 & 1.054 & 32646.16 & 32646.06 & 0.10 & $6.24 \mathrm{E}+07$ & $74.50 \%$ & $36.22 \%$ \\
\hline
\end{tabular}

